Debate / Controversy

\title{
Entre recalibración y continuidad: el contexto del nacimiento del IMV
}

\author{
Between recalibration and continuity: the context of the birth of \\ the $\mathrm{MVI}$
}

\author{
Ana Arriba González de Durana \\ Universidad de Alcalá, España \\ ana.arriba@uah.es \\ Manuel Aguilar-Hendrickson \\ Universidad de Barcelona, España \\ manuel.aguilar.hendrickson@ub.edu \\ Recibido / Received: 08/09/2020 \\ Aceptado / Accepted:22/01/2021

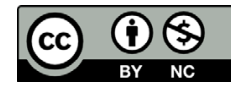

\section{RESUMEN}

España, a diferencia de otros países meridionales, recalibró desde los años 1980 su sistema de garantía de ingresos, conteniendo el nivel contributivo y creando un conjunto de programas asistenciales. A pesar de su significativo impacto, los límites e incoherencias de ese conjunto asistencial explican que, desde 2015, su reforma esté en la agenda política nacional. Aunque el conjunto existente tiene mayor impacto que sus equivalentes italiano o griego, su diseño está aún anclado en una concepción «fordista» que asocia empleo con seguridad económica. Este modelo se muestra insuficiente para hacer frente a las transformaciones de la estructura de los hogares, a las dificultades de acceso a la vivienda, pero sobre todo al crecimiento de formas precarias de participación en el empleo. El artículo analiza la trayectoria del conjunto de la garantía de ingresos en el que el nuevo Ingreso Mínimo Vital mejora apreciablemente la cobertura asistencial, pero deja sin resolver la adecuación del conjunto a las nuevas formas de empleo.

Palabras clave: Rentas mínimas, Asistencia Social, Ingreso Mínimo Vital, Estado de bienestar español, Pobreza

\section{ABSTRACT}

Spain, unlike other Southern European countries, recalibrated its income maintenance system in the 1980s, containing its contributory programs and creating a set of means-tested programs. Despite its contribution to severe poverty reduc-tion, this set has limitations and inconsistencies that have led to its reform entering the national political agenda since 2015. These programs, although more effective than their Italian or Greek equivalents, are still very much linked to a Fordist model that expects any job to be able to lift a household out of poverty. This model is unable to cope with changes in family structure, increasingly difficult access to housing and, above all, new forms of precarious employment. The article analyses the path of those guaranteed income schemes in which the new Minimum Living Income benefit may improve the coverage of existing social assistance programs but leaves unresolved its adequacy to the new forms of employ-ment.

Keywords: Minimum income, Social assistance, Minimum Living Income, Spanish welfare state, Poverty 


\section{INTRODUCCIÓN}

La política de renta mínima estuvo ausente casi por completo de la agenda política central desde los años 1980 hasta 2015. Parecía aceptarse que se trataba de un asunto de responsabilidad autonómica que no requería de encaje con otras políticas de prestación no contributiva sujeta a un límite de renta, es decir, de garantía asistencial de ingresos. Sin embargo, desde las elecciones generales de 2015, el asunto ha estado presente en los programas de los principales partidos, con una amplia variedad de propuestas, desde la coordinación de las rentas mínimas autonómicas hasta la creación de un complemento salarial fiscal (crédito fiscal estilo Earned-Income Tax Credit, EITC), pasando por varias versiones de renta mínima, además de una iniciativa legislativa popular promovida por los sindicatos mayoritarios. La resaca de la Gran Recesión y la discreta, pero firme, presión de las instituciones europeas parecen algunos de los principales factores que explican esta irrupción en la agenda política.

El nacimiento del Ingreso Mínimo Vital (IMV) en junio de 2020 parece estar cargado de paradojas y contradicciones. El gobierno previó un proceso lento, que comenzaría por una mejora de las Prestaciones Familiares por Hijo/a a Cargo (PFHC) y culminaría en algún tipo de renta mínima al final de la legislatura (Partido Socialista Obrero Español y Unidas Podemos, 2016), pero se ha materializado en un brevísimo período de tiempo en una renta mínima y la supresión de la mayoría de las PFHC. La pandemia y la urgencia pueden explicar esta aceleración, aunque el IMV se ha concebido como un programa «estructural» más que como una respuesta de emergencia. Durante su gestación llovieron críticas bastante duras contra el proyecto ${ }^{1}$, pero el decreto ley se convalidó sin votos en contra, con la única abstención de los diputados de Vox. Se ha presentado como una medida estudiada en detalle y profundidad, cuando el breve tiempo de su gestación ni siquiera ha permitido una negociación calmada para encajarlo con las rentas mínimas autonómicas. La rapidez de su diseño y aprobación contrastan con una implementación más lenta y complicada de lo anunciado.

En este artículo abordamos el contexto general de las reformas de la garantía de ingresos para la población potencialmente activa en España desde los años 1980, sus logros y sus limitaciones, para intentar situar el nacimiento del IMV ${ }^{2}$ desde una perspectiva amplia en el largo plazo.

\section{¿LA PIEZA QUE FALTA A LOS ESTADOS DE BIENESTAR MEDITERRÁNEOS?}

A los Estados de bienestar mediterráneos se les atribuye con frecuencia el defecto de haber sido capaces de desarrollar un nivel de protección contributivo bastante sólido, pero carecer de un nivel asistencial suficiente. Mientras los estados continentales o bismarckianos combinan un nivel contributivo para insiders con un nivel asistencial menor pero suficiente para outsiders, en los países del Sur el menor gasto total en protección social habría sido "capturado» por los insiders (con «excesos de generosidad» dificiles de justificar) a costa de dejar desprotegidos a los outsiders. Ello explicaría su limitada capacidad de reducir la pobreza extrema, la captura de las políticas sociales por los trabajadores (en especial varones) y los jubilados a costa de la infancia y las familias (en especial en sus funciones de cuidado que recaen sobre todo en las mujeres) (Ferrera, 1996, 2005; Gough, 1996; Marí-Klose y Moreno-Fuentes, 2013; Mingione y Benassi, 2019). Una crítica análoga se dirige a la protección

\footnotetext{
1 Las críticas provenían tanto de posiciones ideológicas de izquierdas como de derechas. Los partidos políticos de derecha calificaron la medida de ser un pago clientelar y populista (el término empleado por los miembros de Vox fue "paguita») o una medida innecesaria (ya existían en las CCAA) y creadora de desempleo. Desde posiciones ideológicas de izquierda se valoró como insuficiente («de miseria»), focalizada en la pobreza y condicionada (en especial porque está vinculada a la realización de actividades de inclusión).

2 Las luces y sombras del diseño e implementación del IMV han sido recientemente objeto de análisis a corto plazo, que han aparecido en forma de post en Blogs colaborativos como Llei d'Engel (http://lleiengel.cat/), Agenda Pública (http://agendapublica.elpais.com) o Nada es Gratis (https://nadaesgratis.es/). La información del Ministerio de Inclusión, Seguridad Social y Migraciones está disponible en https://revista.seg-social.es/category/imv/.
} 
del empleo, denunciando una combinación perversa de «sobreprotección» de los estables y precariedad extrema de los outsiders.

Se trataría de una especie de «fordismo congelado» (Esping-Andersen, 1996; Palier y Martin, 2007) en el que ciertos grupos de trabajadores y jubilados privilegiados estarían bloqueando, con su poder sindical y electoral, las reformas necesarias. En el caso de las políticas de mantenimiento de ingresos, la «recalibración pendiente» pasaría por reducir los «excesos de generosidad» de la protección contributiva para mejorar la protección asistencial en forma de renta mínima (orientada a promover la activación) y mejora de las prestaciones por hijos. Las recomendaciones de las instituciones europeas durante el último decenio han insistido en ambos elementos (Comisión Europea, s. f.). Recomendaciones que, en países como Grecia, han adquirido la forma de imposiciones ante las dilaciones del gobierno griego en la creación de una renta mínima (Aguilar-Hendrickson y Arriba, 2020a; Jessoula y Natili, 2020; Matsaganis, 2020; Pereirinha, Branco, Pereira y Amaro, 2020).

\section{LA RECALIBRACIÓN DE LOS AÑOS 1980}

Sin embargo, nuestra interpretación del caso español matiza esta descripción del esquema protector mediterráneo. España realizó una serie de reformas en profundidad de sus sistemas de mantenimiento de ingresos (pensiones, desempleo $\mathrm{y}$, en menor medida renta mínima y prestaciones familiares) entre finales de los años 1970 y principios de los 1990 (Rodríguez-Cabrero, 1993, 2011; Aguilar-Hendrickson y Arriba, 2020b). Dichas reformas supusieron una recalibración muy sustancial de las políticas de garantía de rentas.

Las reformas redujeron la "generosidad» del nivel contributivo, y corrigieron algunas disfunciones del sistema. En el campo de las pensiones contributivas, las reformas ampliaron de 10 a 15 los años cotizados exigibles para la jubilación, y aumentaron de 2 a 8 y "posteriormente" a 15 los años para el cálculo del importe de la pensión. También introdujeron una cuantía máxima, que en 1986 equivalía a 4,7 veces el salario mínimo y en la actualidad equivale a 2,9 veces, cuyo efecto es una erosión de la tasa de reemplazo para las personas con ingresos medios y altos (Conde-Ruiz y González-Martínez, 2014). En el campo del desempleo contributivo, las reformas de 1980-1984 y de 1992 endurecieron el acceso a las prestaciones, limitaron su duración y redujeron su importe en relación con el salario anterior (Cruz-Roche, 1994; Pino y Ramos, 2009).

Las reformas también crearon un nivel de protección asistencial prácticamente inexistente con anterioridad. En el terreno de las pensiones, se crearon los complementos de mínimos (una «renta mínima» para pensionistas) y las pensiones no contributivas. En el campo del desempleo, se consolidó un conjunto de subsidios asistenciales, dirigidos a personas desempleadas con determinadas características. Las PFHC, cuya cuantía estaba congelada desde principios de los años 1970, se actualizaron y focalizaron en los hogares de bajos ingresos, aunque sus cuantías volvieron a quedar congeladas durante años.

Este conjunto de programas asistenciales se desarrolló durante los años 1980 y primeros 1990 desde el nivel de gobierno central. En un proceso separado y en ocasiones en conflicto con las reformas centrales, entre 1988 y 1995 se establecieron rentas mínimas en las 17 comunidades autónomas (en adelante RMAs). Se trata de un conjunto de programas que presentan grandes diferencias en cuanto a su diseño, su amplitud y su impacto, que van desde rentas mínimas amplias de tipo generalista hasta programas muy focalizados y de alcance limitado. Su desarrollo se hizo en el marco de la competencia autonómica en «asistencia social» (Aguilar-Hendrickson, Gaviria y Laparra, 1995).

Estas transformaciones permiten hablar de una recalibración de los programas de garantía de ingresos que puede calificarse de «asistencialización» (Rodríguez-Cabrero, 
1993). Esta reforma supuso un ajuste de los programas contributivos y una expansión de los asistenciales, con el fin de corregir el sesgo en beneficio de los insiders. Así se configuró un «nivel» o modelo asistencial particular, que con el tiempo ha mostrado algunas limitaciones importantes. En lo que se refiere a los programas dirigidos a la población potencialmente activa, el modelo se caracteriza por:

a) Una clara separación de programas dirigidos a personas desempleadas con bajos ingresos y a personas en situación de exclusión social, que coincide con la divisoria entre nivel asistencial del desempleo (responsabilidad central) y la asistencia social (responsabilidad autonómica).

b) La vinculación del mayor de estos dos componentes (el desempleo asistencial) a la condición de persona desempleada, que excluye por tanto a las personas con empleo con muy bajos ingresos. Algunas rentas mínimas autonómicas están vinculadas a la condición de desempleado, mientras que otras focalizan en función del nivel de ingresos, con independencia de la situación laboral de la persona perceptora.

Esta separación formal entre desempleados y «excluidos sociales», así como la exclusión de las personas con empleo de la protección asistencial responde a la visión "fordista» prevalente en aquel período, según la cual las personas con empleo tendrían «resuelto» el acceso a unos ingresos suficientes. Estas reformas se preocupan más por las primeras formas de precariedad laboral «postfordista» relacionadas con la discontinuidad temporal del empleo que por las vinculadas a empleos de baja intensidad (en horas o jornadas) pero más continuados en el tiempo.

La distribución del gasto en prestaciones de garantía de ingresos durante la pasada década (Tabla 1) muestra un sistema en el que las prestaciones contributivas, en especial las pensiones, son las grandes protagonistas. Esto sería coherente con un modelo de Estado de bienestar mediterráneo. Sin embargo, observamos también que las prestaciones asistenciales tienen una presencia limitada pero relevante. Esto es así sobre todo para las prestaciones asistenciales dirigidas a población potencialmente activa. En el periodo de recuperación económica y del empleo 2012-2018, disminuyó el volumen de gasto destinado a los subsidios de desempleo, pero en menor medida que el gasto en prestaciones contributivas. Es más significativo aún el crecimiento del gasto en rentas mínimas autonómicas a las que pueden acceder personas desempleadas que carecen de o han agotado las prestaciones o subsidios de desempleo. 
Tabla 1. Gasto (millones de euros corrientes) en prestaciones de garantía de ingresos (contributivas, asistenciales y universales) en España en 2012, 2015 y 2018

\begin{tabular}{lccc}
\hline Contributivas & $\mathbf{2 0 1 2}$ & $\mathbf{2 0 1 5}$ & $\mathbf{2 0 1 8}$ \\
\hline Pensiones contributivas & 95.736 & 107.789 & 120.927 \\
Prestación por desempleo & 22.627 & 12.973 & 11.704 \\
Universales & & & \\
Prestación por hijo a cargo con discapacidad & 981 & 1.069 & 1.169 \\
Asistenciales & & & \\
Complementos de mínimos de pensiones & 7.473 & 7.280 & 7.142 \\
Pensiones no contributivas & 2.256 & 2.371 & 2.465 \\
Subsidios por desempleo & 9.052 & 7.583 & 6.648 \\
Rentas mínimas autonómicas & 855 & 1.360 & 1.520 \\
Prestación por hijo a cargo sin discapacidad & 284 & 343 & 346 \\
& & & \\
Total de prestaciones asistenciales & 19.919 & 18.936 & 18.121 \\
Total de prestaciones contributivas y universales & 119.343 & 121.831 & 133.800 \\
\hline
\end{tabular}

Fuente: Estadísticas de la Seguridad Social, IMSERSO y Comunidades Autónomas

\section{¿HA FUNCIONADO EL MODELO?}

Este conjunto de programas asistenciales ha sido criticado con frecuencia por su fragmentación, limitado alcance y escaso impacto en la reducción de la pobreza. Aunque estas críticas están justificadas, una comparación con algunos países continentales y del Sur de Europa hace pensar que el caso español no se alinea plenamente con los demás.

La Figura 1 muestra el impacto de las pensiones, por un lado, y de otras prestaciones económicas (desempleo, familia, asistencia social, vivienda) en la reducción de la pobreza grave (inferior al 40 por ciento de los ingresos medianos) entre los menores de 65 años. ${ }^{3}$

3 Usamos el riesgo de pobreza grave (umbral 40 por ciento de la mediana) porque las prestaciones asistenciales tienen importes más cercanos a ese umbral que al habitualmente utilizado (60 por ciento de la mediana). Aunque el dato se refiere a menores de 65 años, en sus hogares puede haber personas mayores de 65 , y parte de la contribución de las pensiones a la reducción de la pobreza viene de ahí. 
Figura 1. Población menor de 65 años en situación de riesgo de pobreza monetaria grave (umbral: 40 por ciento de la mediana equivalente de ingresos) antes y después de transferencias sociales. Italia, Grecia, Alemania, Francia y España, 2004-2018.
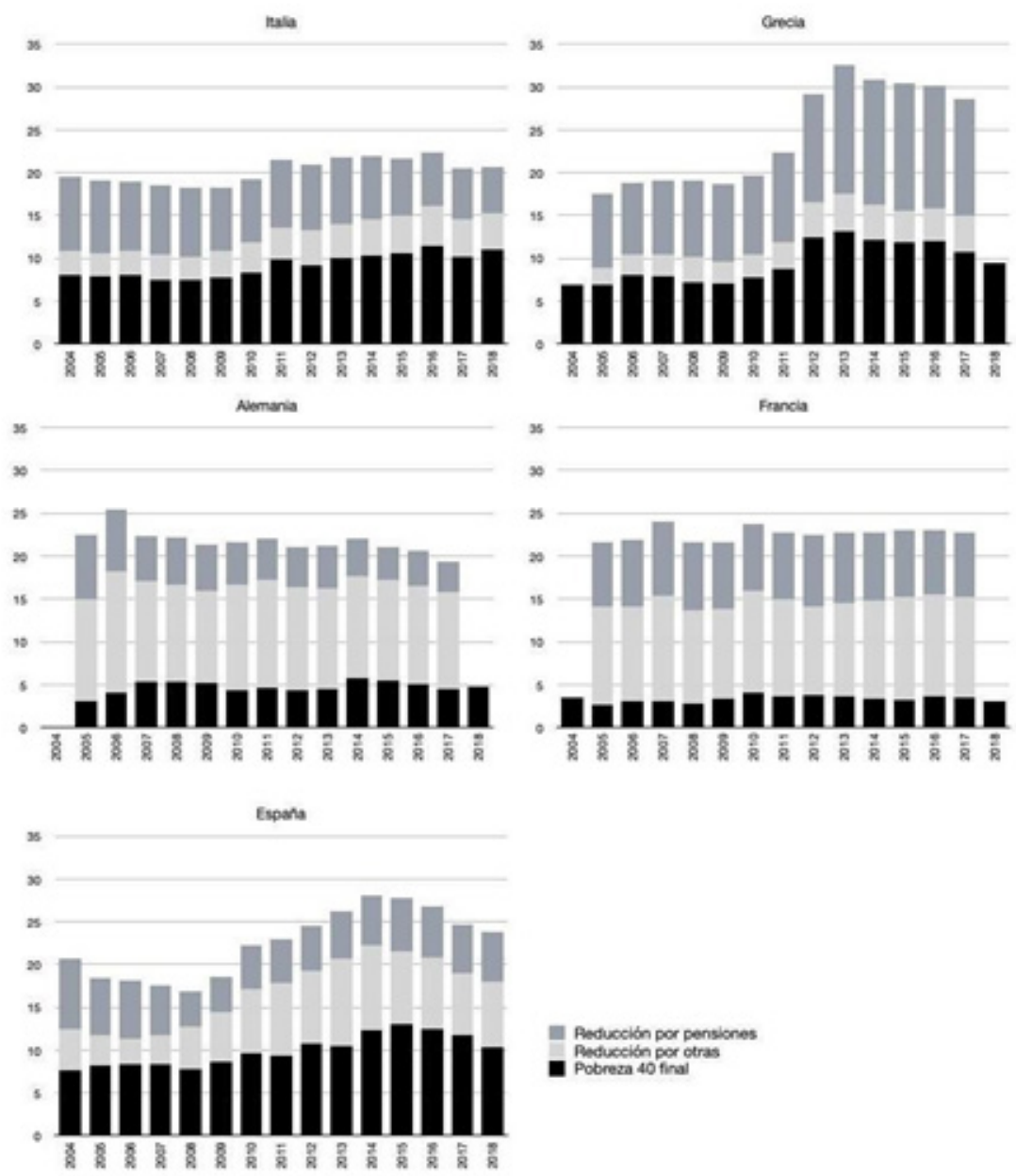

Fuente: Eurostat EU-SILC

En Italia y, sobre todo, en Grecia las pensiones contribuyen significativamente a la reducción de la pobreza grave de los menores de 65 años, mientras que las demás prestaciones contribuyen muy poco. En Francia y Alemania, países continentales típicos, las "otras prestaciones» tienen mayor papel en la reducción de la pobreza grave de los menores de 65 años, mientras que las pensiones tienen un papel mucho menor.

El caso español no se alinea con claridad con ninguno de los dos modelos. El riesgo de pobreza monetaria grave de los menores de 65 antes de transferencias aumentó significativamente durante la Gran Recesión, con una intensidad sólo superada por Grecia. Sin embargo, ese aumento se vio bastante corregido por la expansión de las "otras prestaciones» (principalmente las de desempleo), cuyo papel es muy superior al de las pensiones para la población menor de 65 años. La comparación es más evidente en la Figura 2 (con datos de 2012). 
Figura 2. Reducción del riesgo de pobreza monetaria grave (umbral: 40 por ciento de la mediana equivalente de ingresos) según transferencias sociales (pensiones y otras).

Población menor de 65 años. Italia, Grecia, España y Portugal, 2012.

20

15

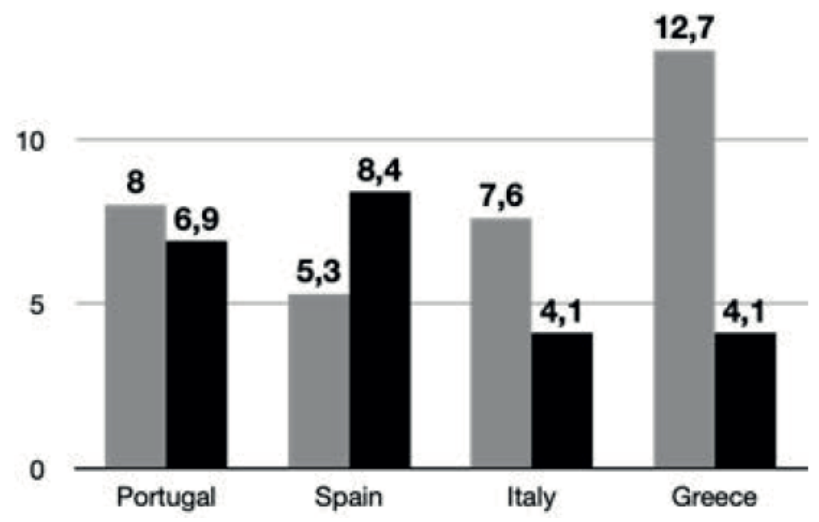

Reducción por pensiones

Reducción por otras

Fuente: Eurostat EU-SILC

El papel central de las prestaciones por desempleo (contributivas y asistenciales) en la reducción del riesgo de pobreza grave en España parece coherente con la forma en la que nuestro mercado de trabajo gestiona los ajustes. El mercado de trabajo español es conocido por su tendencia a destruir empleo a gran escala en las situaciones de crisis. Al menos hasta 2020, en las situaciones dificiles ha prevalecido la flexibilidad externa, que comienza por la no renovación de los contratos temporales y con frecuencia recurre al despido de los permanentes. Esta flexibilidad externa rompe el vínculo entre empresa y persona empleada de forma bastante definitiva, y se traduce en la eliminación prácticamente total de los ingresos de las personas afectadas. Este modelo produce una pérdida drástica de ingresos para las personas que quedan sin empleo, en ocasiones compensadas en el conjunto del hogar por la presencia de alguna otra persona con empleo (o pensión). El modelo de garantía de ingresos ligado a la condición de persona desempleada tiene una capacidad apreciable de sustituir (parcialmente) los ingresos perdidos, siempre que la persona se mantenga en situación de desempleo.

Hay una cierta "coherencia» en que un país que se ajusta destruyendo empleo a gran escala responda sobre todo con prestaciones por desempleo. Pero esta respuesta deja prácticamente sin protección a las personas que tienen algunos ingresos derivados de empleos de baja intensidad. Este problema se traduce simultáneamente en una desprotección (que seguramente explica buena parte de la pobreza grave que se mantiene en períodos de crecimiento) y en un desincentivo al empleo (o al empleo legal y de corta duración). Este es, seguramente, uno de los puntos débiles de este modelo de garantía de ingresos. 


\section{LOS LÍMITES DEL MODELO Y LAS VÍAS DE REFORMA}

Las limitaciones de este modelo de garantía asistencial de ingresos se han hecho más patentes con las transformaciones sociales producidas desde los años 1980. La creciente fragmentación del empleo y el desarrollo de formas de precariedad que ya no se limitan al empleo a tiempo completo de duración determinada, la extensión de las situaciones de precariedad laboral y desempleo hacia los sustentadores principales (relativamente a salvo en los años 1980), la reducción del tamaño de los hogares y el encarecimiento de la vivienda han contribuido a poner contra las cuerdas este modelo. Las principales limitaciones son:

a) La «focalización» de los programas es heterogénea. Los umbrales de ingresos para poder percibir las diversas prestaciones asistenciales son diferentes y se calculan de forma distinta (Ayala, Arranz, García-Serrano y Martínez-Virto, 2016).

b) La dualidad interna del conjunto asistencial, que separa radicalmente desempleo y «exclusión social» en programas diferentes dependientes de administraciones distintas, cuando los límites entre ambas situaciones son cada vez más difusos.

c) La exclusión, completa en el caso del desempleo asistencial y extendida en una parte de las rentas mínimas autonómicas, de las personas con empleo, aunque pertenezcan a hogares de muy bajos ingresos.

d) Aunque las evaluaciones son limitadas, hay razones para pensar que la oferta de acciones de apoyo a la incorporación laboral y social es insuficiente y de dudosa eficacia.

Estas limitaciones explican la aparición de una serie de propuestas de reforma en los últimos años (Noguera, 2019). Varias de ellas buscan extender la protección a los «trabajadores pobres», con mayor o menor voluntad de cerrar la brecha entre asistencia social y desempleo asistencial, estableciendo la compatibilidad de empleo y prestaciones y mecanismos de incentivación al acceso al empleo (descuento parcial de los ingresos por trabajo, crédito fiscal). En esa línea se han situado las siguientes:

a) La propuesta de Ciudadanos de un «complemento salarial», inspirado en programas como el EITC estadounidense, supondría la creación de un programa separado para las personas de ingresos bajos, pero con empleo. No concretaba los cambios que conllevaría en las rentas mínimas ni en los subsidios por desempleo.

b) La propuesta de Podemos de creación de una renta mínima garantizada de baja condicionalidad y nivel relativamente alto, que tendería a absorber (sin mención explícita) buena parte de los subsidios por desempleo, unificando el espacio desempleo asistencial/asistencia social, y dejando a las comunidades autónomas un papel de complementar la prestación estatal.

c) La propuesta de un Ingreso Mínimo Vital (con el mismo nombre pero diferente en algunos aspectos a la propuesta de IMV del PSOE) de la Autoridad Independiente de Responsabilidad Fiscal (AIReF, 2019), tras evaluar la propuesta sindical de ingreso mínimo. Es la que de forma más explícita tendería a integrar renta mínima y desempleo asistencial (o al menos una parte de éste).

d) Las diversas propuestas de renta básica universal (Arcarons, Raventós y Torrens, 2017; Gómez-Frías y Sánchez-Chaparro, 2020), aunque con menor impacto en el debate político, conllevan también una compatibilidad con el empleo y la integración de un buen número de prestaciones, en especial, asistenciales.

Otras propuestas mantienen la separación entre desempleo asistencial y asistencia social: 
e) La propuesta sindical (presentada como una iniciativa legislativa popular en 2015) de creación de una prestación de ingresos mínimos que establecía una incompatibilidad casi completa con el empleo. La propuesta supondría una generalización del subsidio asistencial por desempleo a la práctica totalidad de los desempleados con ingresos muy bajos, pero sin entrar en el terreno de la mejora de los ingresos de las personas con empleo e ingresos modestos.

f) La propuesta inicial de IMV del PSOE ponía el énfasis en una mejora sustancial de las PFHC, a la que seguiría una renta mínima estatal cuyos detalles no estaban plenamente perfilados (PSOE, 2016). Su esbozo inicial como prestación de cuantía fija se alejaba de los modelos dominantes de renta mínima. Sin embargo, la propuesta se había dejado bastante abierta a un debate con las comunidades autónomas a lo largo de la legislatura.

\section{EL INGRESO MÍNIMO VITAL DE 2020}

En junio de 2020 el gobierno central creó el IMV. Lo aprobado es una reforma mucho más limitada que la planteada por la Autoridad Independiente de Responsabilidad Fiscal (AIReF) y que las líneas marcadas en el acuerdo de gobierno de coalición. Las dificultades presupuestarias y la urgencia parecen haber influido en este cambio. La AIReF estimaba un coste global de unos 5.500 millones de euros, que podrían reducirse con un ahorro de unos 2.000 procedentes del gasto actual en otras prestaciones (Renta Activa de Inserción (RAI), algunos subsidios por desempleo) (AIReF, 2019). Estas estimaciones tal vez fueran algo optimistas, y hay otras estimaciones que apuntaban a costes más elevados para una prestación de características parecidas (Fernández et al., 2015). El límite asumido de unos 3.000 millones y la supresión de las PFHC han supuesto la opción por un modelo más limitado.

El diseño ha situado al IMV 2020 casi exclusivamente en el espacio social ocupado (con una cobertura incompleta) por las rentas mínimas autonómicas. Establece un suelo mínimo de protección en el campo de las RMAs (Arriba y Rodríguez-Cabrero, 2021). Es probable, como ya apuntan los primeros datos disponibles 4 , que el IMV absorba buena parte de las RMAs, elevando la baja cobertura en bastantes comunidades, aunque está por ver el efecto de las discrepancias en la fórmula de cómputo de los ingresos entre el IMV y las RMAs. Parece menos probable que «conecte» los espacios institucionales del desempleo asistencial y la asistencia social (cuyos espacios «sociales» hace tiempo que están integrados). En este sentido, la opción por un IMV limitado ha hecho entrar a la Seguridad Social no contributiva plenamente en el espacio de la asistencia social autonómica sin modificar en lo fundamental la estructura preexistente. No es descartable que esto se pueda traducir en un desplazamiento o expulsión (crowding out) de una parte de la acción autonómica en este campo.

La desaparición de las PFHC supone la pérdida de una protección a la que podían acceder los hogares en situación de pobreza que tienen ingresos superiores al umbral establecido. Se amplía así la brecha entre los hogares que acceden a la protección para sus hijos a través del IMV y aquellos hogares con niños que acceden a las exenciones, reducciones y deducciones fiscales.

\footnotetext{
4 Los datos iniciales publicados por el Ministerio de Inclusión, Seguridad Social y Migraciones muestran que el IMV, en marzo de 2021, supera con creces la cobertura de la renta mínima en comunidades como Andalucía, Castilla-La Mancha, Comunidad Valenciana, Murcia y Ceuta y Melilla, mientras sigue siendo muy inferior a la renta mínima en Cataluña, Asturias, Baleares, Cantabria o La Rioja.
} 


\section{CONCLUSIONES}

La recalibración de los años 1980 dio origen a un nivel de garantía asistencial de ingresos de cierta entidad, aunque con una clara prioridad por la población inactiva y, en segundo lugar, por la desempleada, y con un alcance muy limitado en cuanto a su impacto sobre las familias con menores, las personas pobres con empleo y los "excluidos sociales". Este nivel, aunque fragmentado, desigual y de alcance limitado ha tenido un impacto apreciable en la reducción de las formas extremas o graves de pobreza. Este rasgo diferencia el caso español de otros países del Sur con un nivel asistencial menos desarrollado.

El diseño de estos mecanismos de garantía asistencial de ingresos está marcado por una organización social «fordista» en la que la participación en el empleo garantizaba protección frente a la pobreza. Supuso una adaptación a la primera forma de precariedad laboral, la del empleo temporal. La generalización de formas más fragmentadas de empleo diluye la diferenciación entre empleo, desempleo y exclusión social sobre las que se asienta el modelo asistencial español y muestra sus limitaciones (desprotección e incentivos inadecuados).

El diseño del IMV tiene la virtud de plantear un suelo común para la protección de las situaciones más extremas de pobreza que puede ser complementado por las rentas autonómicas. Sin embargo, en su configuración inicial, no parece superar la distinción entre las poblaciones en situación de necesidad, entre las personas en situación de desempleo y los hogares en riesgo de exclusión.

\section{FINANCIACIÓN}

Este trabajo se ha realizado en el marco de los proyectos "INCOME-INN: Innovaciones en programas de garantía de rentas: políticas, pilotos y propuestas" (referencia PID2019107589GB-100) y “PECOTSAS: Perspectiva comparada del Tercer Sector de Acción Social en las Comunidades Autónomas (CSO2017-85486-P)" financiados por el Ministerio de Ciencia e Innovación dentro del Plan Nacional de I+D+i.

\section{REFERENCIAS}

Aguilar-Hendrickson, M., y Arriba, A. (2020a). The politics of safety net reform in Southern Europe during the Great Recession: Introduction to the regional issue. Social Policy and Administration, 54 (4), 533-538. https://doi.org/10.1111/spol.12609

Aguilar-Hendrickson, M., y Arriba, A. (2020b). Out of wilderness? The coming back of the debate on minimum income in Spain and the Great Recession. https://doi.org/10.1111/ spol.12605

Aguilar-Hendrickson, M., Gaviria, M. y Laparra, M. (1995). La caña y el pez. Estudio sobre los salarios sociales de las Comunidades Autónomas. Ma-drid: FOESSA.

Autoridad Independiente de Responsabilidad Fiscal (2019). Los programas de rentas mínimas en España. Estudio. Madrid: AIReF.

Arcarons, J., Raventós, D. y Torrens, L. (2017). Renta básica incondicional. Una propuesta de financiación racional y justa. Barcelona: Ediciones del Serbal.

Arriba, A. y Rodríguez-Cabrero, R. (2021). "New developments in the national guaranteed minimum income scheme in Spain", ESPN Flash Report 2021/15. https:// bit.ly/3plS2kr 
Ayala, L., Arránz, J. M., García-Serrano, C. y Martínez-Virto, L. (2016). El sistema de garantía de ingresos mínimos en España: tendencias, resultados y necesidades de reforma. Recuperado de https://www.mscbs.gob.es/ssi/familiasInfancia/ServiciosSociales/ EstudiosNacionales/SistemGarantIngresosEnEsp.pdf

Comisión Europea (s. f.). European Semester. Country Specific Recommendations. Recuperado de https://ec.europa.eu/info/business-economy-euro/economicand-fiscal-policy-coordination/eu-economic-governance-monitoring-preventioncorrection/european-semester/european-semester-timeline/eu-country-specificrecommendations_es

Conde-Ruiz, J. I. y González-Martínez, C. I. (2014). From Bismarck to Beveridge: The other pension reform in Spain. Documentos de trabajo del Banco de España, 1417. Recuperado de https://www.bde.es/f/webbde/SES/Secciones/Publicaciones/ PublicacionesSeriadas/DocumentosTrabajo/14/Fich/dt1417e.pdf

Cruz-Roche, I. (1994). La protección por desempleo. En M. Juárez (dir.), V Informe sociológico sobre la situación social de España. Sociedad para todos en el año 2000 (pp.1.507-1.518). Madrid: FOESSA.

Pino, E. del y Ramos, J. A. (2009). Proceso político y reformas de la protección por desempleo en España 1984-2008. En L. Moreno (ed.), Reformas de las políticas de bienestar en España (pp. 137-170). Madrid: Siglo XXI.

Esping-Andersen, G. (1996). Welfare states without work: The impasse of labour shedding and familialism in Continental European social policy. En Welfare states in transition: national adaptations in global economies (pp. 66-87). Londres: Sage.

Fernández, G., Aguilar, M., Arriba, A., Ayala, L., Sanzo, L. y Zalakain, J. (2015). Hacia un sistema más inclusivo de garantía de rentas en España: diferentes alternativas de desarrollo. Madrid: FOESSA. Recuperado de https://www.caritas.es/producto/haciaun-sistema-mas-inclusivo-de-garantia-de-rentas-en-espana-diferentes-alternativasde-desarrollo/

Ferrera, M. (1996). The 'Southern model' of welfare in Social Europe. Journal of European Social Policy, 6(1), 17-37. https://doi.org/10.1177/095892879600600102

Ferrera, M. (2005). Welfare state reform in Southern Europe: Fighting poverty and social exclusion in Greece, Italy, Spain and Portugal. En Welfare State Reform in Southern Europe: Fighting Poverty and Social Exclusion in Greece, Italy, Spain and Portugal. Londres: Routledge. https://doi.org/10.4324/9780203356906

Gómez-Frías, V. y Sánchez-Chaparro, T. (2020). Entender la renta básica. Barcelona: Gedisa.

Gough, I. (1996). Social assistance in Southern Europe. South European Society and Politics, 1(1), 1-23. https://doi.org/10.1080/13608749608454714

Jessoula, M. y Natili, M. (2020). Explaining Italian "exceptionalism" and its end: Minimum income from neglect to hyper-politicization. Social Policy \& Administration, 54(4), 599613. https://doi.org/10.1111/spol.12608

Marí-Klose, P. y Moreno-Fuentes, F. J. (2013). The Southern European Welfare model in the post-industrial order: Still a distinctive cluster? European Societies, 15(4), 475-492. https://doi.org/10.1080/14616696.2013.835853

Matsaganis, M. (2020). Safety nets in (the) crisis: The case of Greece in the 2010s. Social Policy and Administration, 54(4), 587-598. https://doi.org/10.1111/spol.12614 
Mingione, E. y Benassi, D. (2019). El modelo de bienestar en la Europa del Sur y la lucha contra la pobreza y la exclusión social. Panorama Social, (29), 9-23. Recuperado de https://www.funcas.es/revista/pobreza-y-rentas-minimas-septiembre-2019/

Noguera, J. A. (2019). El debate político sobre la garantía de rentas en España: las propuestas de los partidos y sindicatos. Panorama social, (29), 71-90. Recuperado de https://www. funcas.es/revista/pobreza-y-rentas-minimas-septiembre-2019/

Palier, B. y Martin, C. (2007). Editorial Introduction From 'a Frozen Landscape' to Structural Reforms: The Sequential Transformation of Bismarckian Wel-fare Systems. Social Policy \& Administration, 41, 535-554. https://doi.org/10.1111/j.1467-9515.2007.00571.x

Pereirinha, J. A., Branco, F., Pereira, E. y Amaro, M. I. (2020). The guaranteed minimum income in Portugal: A universal safety net under political and fi-nancial pressure. Social Policy \& Administration, 54(4), 574-586. https://doi.org/10.1111/spol.12604

Partido Socialista Obrero Español (2016). Ingreso Mínimo Vital. Recuperado de https:// www.psoe.es/propuestas/politica-social/ingreso-minimo-vital/

Partido Socialista Obrero Español y Unidas Podemos (2019). Coalición Progresista. Un nuevo acuerdo para España. Recuperado de https://www.psoe.es/mediacontent/2019/12/30122019-Coalición-progresista.pdf

Rodríguez-Cabrero, G. (1993). Integración, asistencialización y exclusión en el Estado de bienestar. En J. Albarracín, D. Anisi, F. Esteve Mora, J. González Calvet, J. F. Martín Seco, P. Montes, y G. Rodríguez-Cabrero (eds.), La larga noche neoliberal. Políticas económicas de los ochenta (pp. 271-283). Madrid/Barcelona: Icaria/Instituto Sindical de Estudios.

Rodríguez-Cabrero, G. (2011). The consolidation of the Spanish welfare state (1975-2010). En A. M. Guillén y M. León (eds.), The Spanish Welfare State in European Context (pp. 17-37). Surrey/Burlington: Ashgate.

\section{NOTA BIOGRÁFICA}

Manuel Aguilar-Hendrickson es Sociólogo y profesor de Política social en la Escola de Treball Social de la Universitat de Barcelona. Investiga sobre políticas de renta mínima y servicios sociales. Ha participado en procesos de puesta en marcha de programas de renta mínima y en la evaluación de diversos programas de servicios sociales. Una relación más detallada está disponible en https://www.researchgate.net/profile/Manuel_Aguilar_ Hendrickson/contributions

Ana Arriba González de Durana es Doctora en Sociología por la Universidad Autónoma de Madrid. En la actualidad es profesora de Sociología en la Universidad de Alcalá. Sus trabajos de investigación se han centrado en las políticas de lucha contra la pobreza, en especial de las rentas mínimas, en el marco de las transformaciones de los estados de bienestar europeos. Una relación más detallada en: https://www.researchgate.net/profile/Ana_Arriba 